Rhode Island College

Digital Commons @ RIC

\title{
Attributes of Effective Nurse Care Managers in Patient-Centered Medical Home
}

Susan Corner

Rhode Island College

Follow this and additional works at: https://digitalcommons.ric.edu/etd

Part of the Nursing Commons

\section{Recommended Citation}

Corner, Susan, "Attributes of Effective Nurse Care Managers in Patient-Centered Medical Home" (2012). Master's Theses, Dissertations, Graduate Research and Major Papers Overview. 162.

https://digitalcommons.ric.edu/etd/162

This Major Paper is brought to you for free and open access by the Master's Theses, Dissertations, Graduate Research and Major Papers at Digital Commons @ RIC. It has been accepted for inclusion in Master's Theses, Dissertations, Graduate Research and Major Papers Overview by an authorized administrator of Digital Commons @ RIC. For more information, please contact digitalcommons@ric.edu. 


\title{
ATTRIBUTES OF EFFECTIVE NURSE CARE MANAGERS IN PATIENT-CENTERED MEDICAL HOME
}

\author{
by
}

\section{Susan Corner}
A Major Paper Submitted in Partial Fulfillment of the Requirements for the Degree of
Master of Science in Nursing
in

The School of Nursing

Rhode Island College

2012 
Thank you to my first reader, Kathy Gremel, for her patience and sense of humor. Thank you to my son, David, for helping me with computer issues even though he is in New York! To my pal, Maryellen Danzer, who is a dear soul and a great support. Thanks to Buzz for all your love and support. 


\begin{abstract}
The burden of chronic disease on our medical system and on society is substantial and adds to the difficulty of managing ever-increasing amounts of patient information as care becomes more complex. It has placed the quality of health care in the United States (US) under scrutiny because of medical errors, lack of coordination, patient frustration and poor outcomes. In response to the many challenges, the Patient-Centered Medical Home (PCMH) model of care was developed and is designed to increase the value of our health care and to improve the experience for the patient. The role of the Nurse Care Manager (NCM) in PCMH to help patients with chronic disease is relatively new, but results in the literature are promising.

In this evaluation study, the literature on effective NCM interventions and attributes is reviewed and summarized. In addition, a small sample of NCMs was interviewed to determine the specific attributes and interventions used by NCMs in PCMH. NCMs are effective in assisting patients in managing chronic disease, engaging and activating the patient, transition of care management, and using electronic medical records (EMR) to track patient outcomes. Collaboration with other clinicians, evidence-based and advanced protocol interventions, advanced education and training, and understanding the social and environmental context of the patient were all found to improve patient care. The findings from the literature as well as the interviews explore the components of effective nurse care management and the challenges for the NCM as the role evolves.
\end{abstract}




\section{Table of Contents}

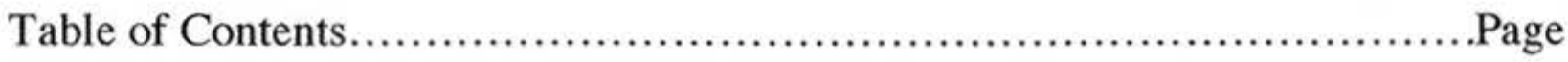

Background/Statement of the Problem..................................... 1

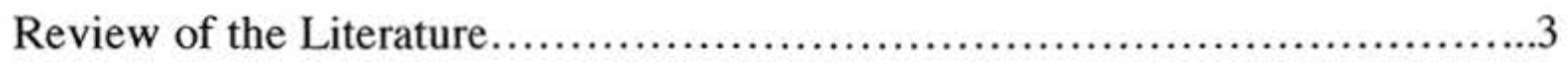

Theoretical Framework........................................................

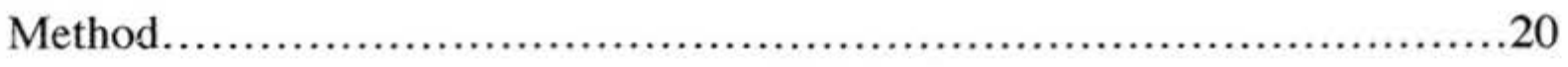

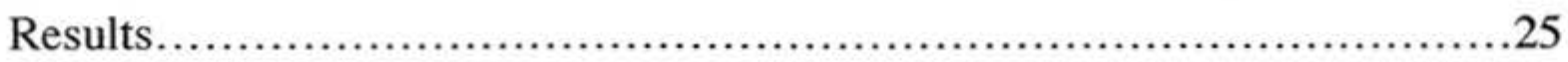

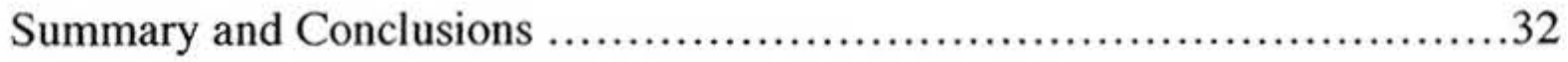

Recommendations and Implications for Advanced Nursing Practice............35

References................................................................

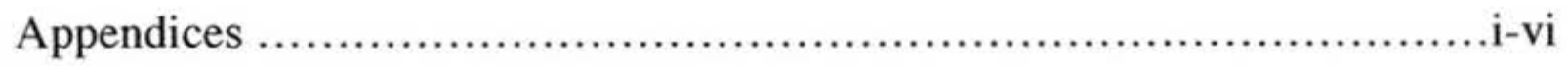


Attributes of Effective Nurse Care Managers in Patient-Centered Medical Home Background/Statement of the Problem

The United States (US) healthcare system needs new innovations to meet the ever-growing burden of chronic disease. Patient care is being delivered in an increasingly uncoordinated fashion, leading to poor outcomes, increased costs, avoidable disability, and premature deaths (Jonas, Kovnar, \& Knickman, 2008). This leads to errors in medication reconciliation and dangerous side effects can result (Jonas, et al., 2008). In this environment, primary care practices struggle to meet the needs of their patients. Ill patients often have to wait to have phone calls returned and to be seen in the office, resulting in unnecessary trips to the Emergency Department as well as avoidable hospital admissions. Follow up on labs and other diagnostic tests as well as referrals to specialists are inconsistent, potentially resulting in the need to repeat tests and causing patient frustration. Patients are also dissatisfied with the lack of timely response and the gaps in coordination of care between the hospital and the primary care providers. Patients are often passive, merely carrying out physician-scripted activities that may or may not reflect their own goals, preferences, and cultures. They often lack the professional support needed to make the changes in lifestyle that would help them to better manage chronic illnesses. In addition, the current pay for service model of reimbursement does not support or provide the motivation for practices to improve outcomes (Bohmer \& Lee, 2009). Clearly, a transformation in the way care is delivered is needed. According to Bohmer and Lee, it is possible to help improve patients' health while reducing costs. 
The National Committee for Quality Assurance (NCQA) has initiated a model for quality practice transformation called the Patient Centered Medical Home (PCMH). It is a team-based model of care led by a patient's personal physician who provides continuous and coordinated care throughout his/her lifetime to maximize health outcomes (Patient-Centered Medical Home, 2011). The PCMH model has set benchmarks for participating practices, including patient self-management support, improved care coordination, evidence-based guidelines for chronic conditions, and performance reporting and improvement (Bowers, 2010). Patients also will have increased access to information about their test results as well as reliable health information through the practice website, email, and telephone contact with the providers (Bowers). Patient safety and efficiency will be improved through electronic medical records (EMR), which includes a list of current medications and allergies. Coordinated care from hospital to home or facility leads to less uncertainty and a sense of continuity by the patient and their caregivers.

The Nurse Care Manager (NCM) uses the EMR to track patient progress and target those patients needing more intensive intervention (Bowers, 2010). Having a NCM embedded in the primary care practice is an essential part of improved care coordination, improved safety, and better outcomes in the PCMH practice model. The role was recommended by the NCQA to help transform our healthcare delivery system from a traditional, reactive, crisis-oriented model to a more holistic, proactive, preventative and coordinated model of care delivery. The general requirements for a NCM are a B.S.N. with three to five years of experience in community health, public 
health, chronic disease management, community nursing, or case management. Experience working with primary care providers to coordinate care and disease management experience is preferred (RI CSI, 2009).

The NCM is a key member of the PCMH team. Thus, the purpose of this evaluation project was to assess the role of the NCM within Rhode Island (RI)PCMH in order to determine the attributes, best practices, advanced protocols, and resources which contribute to the effectiveness of the role in the primary care setting.

\section{Review of the Literature}

A search of CINHAL and Pub Med using the key words US healthcare, quality improvement, patient-centered medical home, and nurse case management was conducted. Of the 30 research articles reviewed, 13 yielded pertinent data regarding NCM role analysis and attributes of effective nurse care managers. In addition to the research articles, the NCQA reports on PCMH as well as the RI Chronic Care Sustainability Initiatives (CSI) updates had helpful information about the development of collaborative, patient-centered care, and the management of patient information through electronic medical records. Jonas and Kovnar's book on healthcare in the United States, IOM reports, and various other resources were utilized in the literature review.

The literature review is divided into two (2) sections: the US Healthcare System and Nurse Care Managers. The Healthcare System outlines issues with the US healthcare system, healthcare quality, payment reform, and describes the patientcentered medical home model. The Nurse Care Manager section examines the specific attributes and interventions of a NCM. 


\section{The US Healthcare System}

The US healthcare system. The US healthcare system "encompasses a sprawling set of activities and enterprises" (Jonas et al., 2008, p.4) and care is delivered in an often haphazard and non-systematic fashion. The US healthcare system is comprised of the public health system, including behavioral health, and medical care (Jonas et al.). Public health focuses on prevention of illness and promoting activities to keep the population healthy. Examples of public health activities are preventative vaccinations, health education campaigns, and environmental concerns such as air and water health, food safety, and behavioral health programs encouraging people to make healthier lifestyle choices. Together, public health and behavioral health account for a fraction of our healthcare spending.

The medical system, which takes care of people once they become ill, focuses on treatment and cures, but not as much on health promotion initiatives. Therapeutic lifestyle changes such as good nutrition, daily exercise, smoking cessation, and stress reduction have a much greater impact on the well being of the population. However, the US spends at least nine times as much on the care of someone who is ill than on strategies to prevent illness (Jonas et al., 2008).

Healthcare quality. Improving quality has always been important, but more recently it has become a crucial issue. Reports of medical errors, substandard care, and inequities in treatment are generating much needed attention in the medical sector as well as in the media. There are shortages of nurses and primary care physicians in some areas, lack of affordable dental care, inadequate mental health services, and language barriers, 
which contribute to a failing and costly system (Jonas et al., 2008). We need a strengthening of the healthcare workforce, a society-wide focus on healthy lifestyles, and an emphasis on patients taking responsibility for their own health (Jonas et al.). Patients need effective care that is delivered in a timely fashion to avoid unnecessary morbidity and over utilization of Emergency Departments and hospitals (Bowers, 2010).

In 2001, the Institute of Medicine (IOM), an arm of the National Academy of Sciences, published a report called Crossing the Quality Chasm: A New Health System for the $21^{\text {st }}$ Century. This Committee of health care experts determined that sweeping redesign of how care is delivered was imperative (IOM) and labeled the difference in the health care we have in the US and the health care we could have a chasm. The authors pointed out that those patients with common chronic conditions require a multidisciplinary infrastructure to meet their increasingly complex needs (IOM). The six aims for improvement for the patients are that health care should be effective, safe, patient-centered, timely, efficient, and equitable. Some of the innovative ideas currently being implemented in health care delivery came from the Health Care Quality Innovation Fund established as a response to the IOM Report. The most relevant to this project are those using information technologies, applying evidence to health care delivery, and aligning payment policies with quality improvement (IOM, 2006).

In response to the IOM Report, the National Committee for Quality Assurance (NCQA) initiated the Patient Centered Medical Home (PCMH) model (PCMH, 2011). The $\mathrm{PCMH}$ is an innovative program for improving primary care that organizes care around patients, using patient feedback to drive quality improvement. $\mathrm{PCMH}$ is 
distinguished from traditional primary care by the commitment the practice makes to enhance access, the improved tracking of risk factors, and enhanced continuity of care including follow up on tests and care at other facilities (PCMH). Also, there is a focus on the use of health information technology to improve patient care through initiatives related to Meaningful Use (MU). MU means that clinicians are using certified electronic health records (EHR) in a way that can be measured in terms of clinical quality. Examples are e-prescribing, tracking patient outcomes, and exchanging health information to improve clinical quality. Also, Medicaid and Medicare reward and provide incentives in the primary care environment (The PCMH Advisory Committee, 2011) for those using EMR. Redesign of primary care must include a broader set of health professionals in order to move effectively into a new model of healthcare delivery (Homer \& Baron, 2010). The PCMH is a paradigm shift in thinking which requires the practices to radically change patient care delivery. Critical success factors included leadership, personal and organizational relationships, financial incentives and resources, engagement with patients and families, health information technology, competent management, and staff development (Homer \& Baron). Also, to improve outcomes, the fee for service model must be changed, as it is counterintuitive to focusing on higher quality, better access and lower costs (Homer \& Baron).

Payment Reforms and Quality Improvement. The problems which are inherent in a fee for service system have been apparent for some time. If a primary care office is paid only for visits, tests, and procedures, there is little incentive to change the focus of the care on these functions. If telephone follow up for patients who are 
chronically ill reduces patient visits, that results in reduced income in the current model (IOM, 2006). Increasingly, payment policies are being enacted that focus on outcomes rather than on the number of visits in order to encourage and support the paridigm shift and quality improvement (Bohmer \& Lee, 2009). The hospitals also have incentives to reduce readmissions and are communicating with the primary care practice when a patient is admitted in order to enhance continuity and improve transitional care from the hospital to home (Bohmer \& Lee). Acccording to the 2006 IOM Report Rewarding Provider Performance: Aligning Incentives in Medicare, the current Medicare payment system is broken. In this document, it was recommended that the US Department of Health and Human Services (USDHHS) rapidly implement pay for performance in Medicare and that providers be rewarded for an improvement in care coordination across health care settings. It was further stated that providers should be assisted financially in the conversion to electronic health information technology in order to meet the goals of tracking and improving patient outcomes (IOM). These are just some examples of the strategies being tested as the nation struggles with the cost of health care. Other strategies to reduce health care cost and improve quality are the PCMH and the use of NCMs.

PCMH. One of the questions being considered in the literature and in practice is how effective the PCMH model is. The PCMH model was originally used in pediatric primary care offices to improve the quality of care by organizing care around the patients. Because the model was so successful in the pediatric environment, it was adapted for use in adult primary care settings (Patient-Centered Medical Home, 2011). Key features of 
this care model are improving care for chronic conditions and reducing emergency department use, hospitalizations, and re-admissions through strengthened care management and transition planning. Tracking of quality measures through EMR and maintaining disease registries helps providers to deliver care that is customized to the patients' needs (PCMH, 2011). The Patient-Centered Primary Care Collaborative (2010) found that the cost savings that result from the implementation of PCMH are significant. In this in-depth report, the group reviewed the evidence from prospective evaluation studies conducted in the US over the last seven years and found consistent improvements in quality of care, patient experiences, and decreased utilization of emergency departments and hospitals (Patient-Centered Primary Care Collaborative, 2010). Thirteen PCMH interventions, including an emphasis on prevention, improved care coordination, the use of EMR, shared decision making with the patients, and clinical decision support tools were evaluated. The authors concluded that "There is now even stronger evidence that investments in primary care can bend the cost curve, with several major evaluations showing that patient centered medical home initiatives have produced a net savings in total health care expenditures for the patients served by these initiatives" (p.1). Also identified in this report was the assertion that transformation to a PCMH must include good leadership, staff development including NCMs, and the effective use of health information technology. Collaborative relationships between the physicians, nurses, medical assistants and managers, with patients and their families, with other providers and the community are also essential (Homer \& Baron, 2010). The role of a Nurse Case Manager (NCM) is identified as an important factor in successful practices. In this new 
model, the physician does not personally provide all the care, but relies increasingly on the NCM to track data and coordinate needed patient interventions (Homer \& Baron). Nurse Case Managers (NCMs)

Nurse case manager role (NCM). Nurse Case management as defined by the American Nurses Association (ANA,2001) is a dynamic and systematic collaborative approach to provide and coordinate health care services to a defined population. The NCM role is designed to reduce fragmentation and lead to quality, cost effective clinical outcomes (ANA). NCMs are responsible for the evaluation of options available to help meet a patient's health needs through collaboration with the patient and physician in order to achieve improved quality of life (Case Management Society of America, 2002). The role of the NCM is in line with the Institute for Healthcare Improvement's triple aim for healthcare: to improve population health, enhance the patient experience and reduce per capita cost (Managed Care, 2011). Having a NCM in the practice to offer patient self-management support, coordination of care, and track patient outcomes is part of the criteria essential to becoming recognized as a PCMH by the NCQA (PCMH, 2011).

Interventions and work by NCMs. A retrospective, cohort study conducted at a clinic providing services to Alaska Native and American Indian people examined how nurse case management affected outcomes for the diabetic population (Wilson, Curtis, Lipke, Bochenski, \& Gilliland, 2005) by comparing two groups of patients, those with NCMs and those receiving standard physician care. The study also investigated which interventions yielded the best results in order to better prepare NCMs to respond to the growing diabetic population in this region. The case load for each NCM averaged 365 
patients; the types of interventions included referrals for specialty examinations and consultations as well as instructing patients in the area of self-monitoring of blood glucose and medication administration. The patients with NCMs were more likely than those in the control group, who received standard care, to have had annual eye examinations, diet and exercise instruction by a registered dietician, dental and foot examinations, and to self monitor their blood glucose levels. After adjustment for variables such as visits with a dietician and type of intervention, the patients who received $\mathrm{NCM}$ enhanced care had greater reductions in a test that measures the average blood glucose over three months, the Hemoglobin( $\mathrm{Hb}) \mathrm{AlC}$. The results for $\mathrm{HbAlC}$ were -0.52 units with $\mathrm{NCM}$ intervention versus -0.17 units without $(\mathrm{p}<0.006)$. This improvement in the $\mathrm{A} 1 \mathrm{C}$ has been shown in other studies to be associated with decreased comorbidities in the diabetic patient. It was concluded that further improvements in reducing average blood glucose levels might be achieved through the use of standing orders that would empower the NCMs to act independently to improve diabetic care. Recently, the Geisinger Health Plan evaluated the effectiveness of embedded NCMs working in practices to manage transitions of care from the hospital to home or skilled care facilities (Managed Care, 2011). The NCMs are notified of admissions and discharges and manage the critical transition period by anticipating needs and avoiding a gap in care during this period. There are $70 \mathrm{NCMs}$ who manage a population of 70,000 patients in the Geisinger system. The authors reported, on the basis of utilization reviews, a $20 \%$ reduction in readmission to hospital due to improved case management of patients with chronic conditions. Nurses worked with patients to develop a self- 
management action plan, which helped patients take action before they experienced exacerbations of their illnesses; for example, a diuretic protocol might be utilized for patients with heart failure. They also ensured that patients were able to visit their physician within 3-5 days after discharge from the hospital. The incidence of readmission to the hospital is highest in the first 30 days after discharge, so phone calls are used to support the patients' transition to home (Managed Care).

A large, five year randomized control trial called the Diabetic Nurse Case Management and Motivational Interviewing for Change (DYNAMIC) study funded by the National Institutes of Health is underway. The target population includes patients with Type 2 diabetes and motivational interviewing (MI) is used. MI was defined by Rollick, Mason, and Butler (1999) as a collaborative interviewing approach between the patient and the provider that seeks to reduce ambivilence toward behavior change and engage a client's intrinsic motivation to overcome barriers to making positive lifestyle changes. Final outcome data is not yet available, but Dellsega, Gabbay, Durdock, and Martinez-King (2010) examined results from a midpoint focus group conducted with a quality control focus to evaluate how diabetic patients were responding to the added clinical intervention of MI by NCMs. Included in the focus groups were clients 21 years and older with a $\mathrm{HbA} 1 \mathrm{C}$ over $7.5 \%$ who had been enrolled in the study for the prevous six months. The researchers found that there were beneficial health and behavioral outcomes resulting from the combination of diabetic $\mathrm{NCM}$ and $\mathrm{MI}$, as compared to standard care (Dellsaga et al.). Although the total number of participants was only 19, themes that emerged showed promise for the intervention. NCMs using MI provided 
gentle accountability, supported patients who were dealing with depression or denial about their diagnosis, and motivated and empowered those in the intervention toward enhanced self care according to their emotional state of readiness. Patients expressed their appreciation for the empathy shown and described feeling hopeful about improving their health and setting their own goals. Limitations of this study include that the sample size was small, and the NCMs were able to spend a full hour with the patient which is more time than is afforded in a standard visit (Dellsaga et al.). This enhanced NCM intervention could be instrumental in helping providers assist people with diabetes to overcome the clinical inertia that many diabetics experience as they learn to better manage self-care.

A local study is being undertaken by Aquidnick Medical Associates (Bowers, 2010), a RI practice that has become a leader in the conversion to PCMH. The lead physician on the overhaul of the practice structure is Dr David Gorelick, who explained the benefits of partnering with Blue Cross/Blue Shield to become a NCQA recognized practice (Bowers, 2010). The practice had the necessary funding through this partnership to convert to EMR and now can track the data on patients to improve the care they receive. They have embarked on a re-evaluation of patient-provider interactions, clinical care, and patient outcomes. The NCMs can now more efficiently track their diabetics as well as others with chronic conditions in the practice to confirm that those patients are receiving state of the art care for their condition (Bowers).

Attributes of effective NCMs. Attributes of effective NCM practice that were associated with improved patient outcomes in the literature are as follows: collaboration 
among clinicians; specific training in glucose pattern management and other advanced protocols; the use of targeted evidence-based interventions; providing care in the patients social context; the use of highly experienced nurses; the use of motivational interviewing to engage and activate the patient; exceptional communication skills; and advanced education.

Collaboration among clinicians. Collaboration among clinicians leads to better outcomes. A meta-analysis of 29 studies on the effectiveness of NCM with patients who have poorly controlled diabetes, concluded that nurse-led team management was superior to a single clinician in achieving blood glucose control. The rationale provided by the authors was that collaboration among clinicians results in better outcomes (Welch, Garb, Zagarins, Lendel \& Gabbay, 2010) because it was easier to find workable solutions to everyday patient problems while sharing expertise with the physician, the pharmacist and the dietician. This meta-analysis reported that nurse-led case management was associated with a mean reduction in $\mathrm{HbA} 1 \mathrm{c}$ of $0.89 \%(p<0.001)$ as compared to usual care by a physician.

Specific training in glucose pattern management and other advanced protocols. The next NCM attribute is using advanced protocols and specific training to improve patient care. For example, specific training in glucose pattern management combined with the ability of NCMs to intensify medication regimens was found to improve patient outcomes (Watts, Lawrence, \& Kern, 2011). This study was a retropective, observational study of a quality improvement intervention within the Cleveland Veteran Administration health care system which operates 12 community-based outpatient clinics. NCMs were 
involved in an internal quality initiative in which they were trained in a protocol for medication management and analyzing blood glucose patterns by a certified diabetic educator nurse practitioner (NP). One of the barriers identified by the organization was that there is a lack of qualified diabetic NCMs to meet the needs of their diabetic population. The NCMs were mentored in the core principles of diabetic self management and glucose pattern management and were also encouraged to become certified in diabetes education. This study of 3,842 patients revealed that those who had a NCM who had the advanced protocol education improved their $\mathrm{HbAlc}$ to a slightly greater extent than those without a NCM. The mean HbAlc at followup was $9.1 \%$ for the patients with enhanced NCM as compared to $9.4 \%$ for those receiving usual care $(\mathrm{p}<.01)$.

Similarly, a randomized control trial in a large managed care organization revealed that the use of treatment algorithms to intensify glucose management led to improved glycemic control (Aubert et al., 1998) which helps to prevent or delay complications of diabetes. This study, which enrolled 17 Type 1 and 121 Type 2 diabetics in primary care clinics in a large health maintainence organization, found that those cared for by diabetic NCMs had average HbA1c decreases of $1.7 \%$ compared to $0.6 \%$ decreases in $\mathrm{HbA} 1 \mathrm{c}$ for those in usual care $(\mathrm{p}<0.01)$ at the 12 month follow up (Aubert et al.). Seventy-two percent of these patients completed follow-up. Although this study is dated and medication treatment algorithms have changed, it highlights the benefits of reinforcement of meal planning and exercise provided by diabetic NCMs.

The use of targeted evidence-based interventions. Targeted evidence based interventions such as ensuring that evidence based treatment guidelines were being used 
for patients with chronic diseases is another component of effective NCM. In the United Kingdom (UK), a structured review of the literature on improving outcomes for patients with diabetes, COPD, and coronary heart disease was conducted (Sutherland \& Hayter, 2009). The authors measured five health outcomes: objective clinical measurements; quality of life and functionality; patient satisfaction; self-care; and adherence to treatment as evaluated by 18 studies on long term chronic health conditions. It was concluded that when the NCMs used evidence based treatment prompts, the patients were more likely to be receiving the recommended medication and experience improved outcomes (Sutherland \& Hayter, 2009). Advanced Practice Nurses (APN) and certified educators are more prepared to use evidence based interventions such as shared medical appointments which employ peer support and mentoring for patients with chronic illnesses. Watts, Gee, O’Day, Schaub, Lawrence, Aron, \& Kirsch (2009), conducted a program evaluation in order to map the specific skills of the APNs in chronic disease management. Among the skills noted were their specific expertise in chronic disease states, practical guidance in day to day management, a holistic perspective and recognition of the importance of family support. The APNs were involved in template development for the EMRs to assist all clinicians in using targeted interventions and best practices for each patient (Watts et al., 2009).

Providing care in the patients' social context. Also noted in the literature on effective case management was the importance of providing care for patients and their families within the context of their environment. A large military treatment facility was the setting for this study on diabetes NCMs ( Mullen \& Kelley, 2006). The study 
examined the effectiveness of interventions post-discharge, and conducted weekly phone calls for the first six weeks to track blood glucose and to reinforce dietary recommendations. Monthly follow-up as well as walk-in appointments were offered, and subjects were then monitored quarterly for the first year and sent for diabetes selfmanagement education classes. It was found that despite the lack of barriers to achieving glycemic control that exist in a free health care setting, the patients frequently did not complete laboratory work and follow treatment recommendations. Limitations of this study include a small sample size and missing data, but it was concluded that more frequent follow-up and robust self-care education is needed to ensure adherence due to the nature of transient military life (Mullen \& Kelley, 2006).

Use of highly experienced nurses. Another factor in effectiveness of NCMs is that highly experienced nurses who function at the level of an advanced practice nurse (APN) are effective case managers (Sutherland \& Hayter, 2009). Sutherland and Hayter conducted a structured review of the literature on nurse case management of three major chronic diseases. Eighteen articles were subjected to thematic analysis based on improved health outcomes. In the UK, nurse case managers are called the "Specialist Nurse" and they actively identify and intensively monitor individuals with chronic illnesses. They are expert nurses who integrate and coordinate support services, teach self-management, and implement specific interventions such as alerting the patient to signs that are red flags for them with their illnesses (Sutherland \& Hayter, 2009). This element of chronic disease management is consistent with the work by Genrich \& 
Neatherlin, (2001) in which the authors recommended that case managers have a strong clinical knowledge base and are able to work effectively within systems of care.

Use of motivational interviewing to engage and activate the patient. The transtheoretical model for assessing readiness to change combined with motivational interviewing was found to be effective. MI is defined by Rollick, Mason, and Butler (1999) as a collaborative interviewing approach between the patient and the provider that seeks to reduce ambivilence toward behavior change and engage a client's intrinsic motivation to overcome barriers to making positive lifestyle changes. An example of an MI intervention was patients with chronic heart failure in the UK (Brodie, Inoue \& Shaw 2006). Many of these patients do not adhere to prescribed exercise programs and experience a decrease in their quality of life due to limited mobility. The study of 60 patients randomized to standard care, motivational interviewing or both found that patients in the standard care plus motivational interviewing group had quality of life scores that were twice as high as the standard care only patients (Brodie et al., 2006).

Exceptional communication skills. A study on the role of the case manager (Genrich \& Neatherlin, 2001), highlighted the communication skills needed by the NCM. These included the use of health information technology, skill in making presentations, documenting assessments, resolving conflict, networking, negotiation, and group interactions. The authors asserted that the NCM role requires advanced skill in interpersonal relationships, critical thinking, systems of care, management, planning, cost accounting and facilitating change. It is suggested in this analysis that case managers should ideally be Clinical Nurse Specialists (CNS) or baccalaureate prepared nurses, but 
with more specific case management educational preparation to help meet the role requirements. Because this article was written in 2001 , some of the information is dated, but the recommendations for role development, clarification and educational requirements are persuasive.

Advanced education. Advanced educational preparation was recommended in the study by Genrich and Neatherlin (2001) because of the range of qualifications needed to fulfill this important role in the delivery of high quality and cost effective health care. Further development of educational programs that include components of case management competencies is advocated by the authors (Genrich \& Neatherlin).

In summary, the literature review demonstrates the effectiveness of the NCM in PCMH for patients and physicians and the potential benefits in terms of quality and cost savings. Based on the literature, four interventions and eight attributes were identified and discussed in the previous sections. The questions that remain are: What qualities and abilities does a NCM need in a PCMH setting? Secondarily, what are the resources used by the NCMs in RI that are the most helpful in their role? These questions will be explored further in this study.

\section{Theoretical Framework}

The framework that was used to guide this study is the Center for Disease Control and Prevention (CDC) framework for program evaluation (CDC, 2011). This framework was created by the CDC in 1997 as a systematic way to evaluate the merits of public health programs and interventions (Figure 1). 


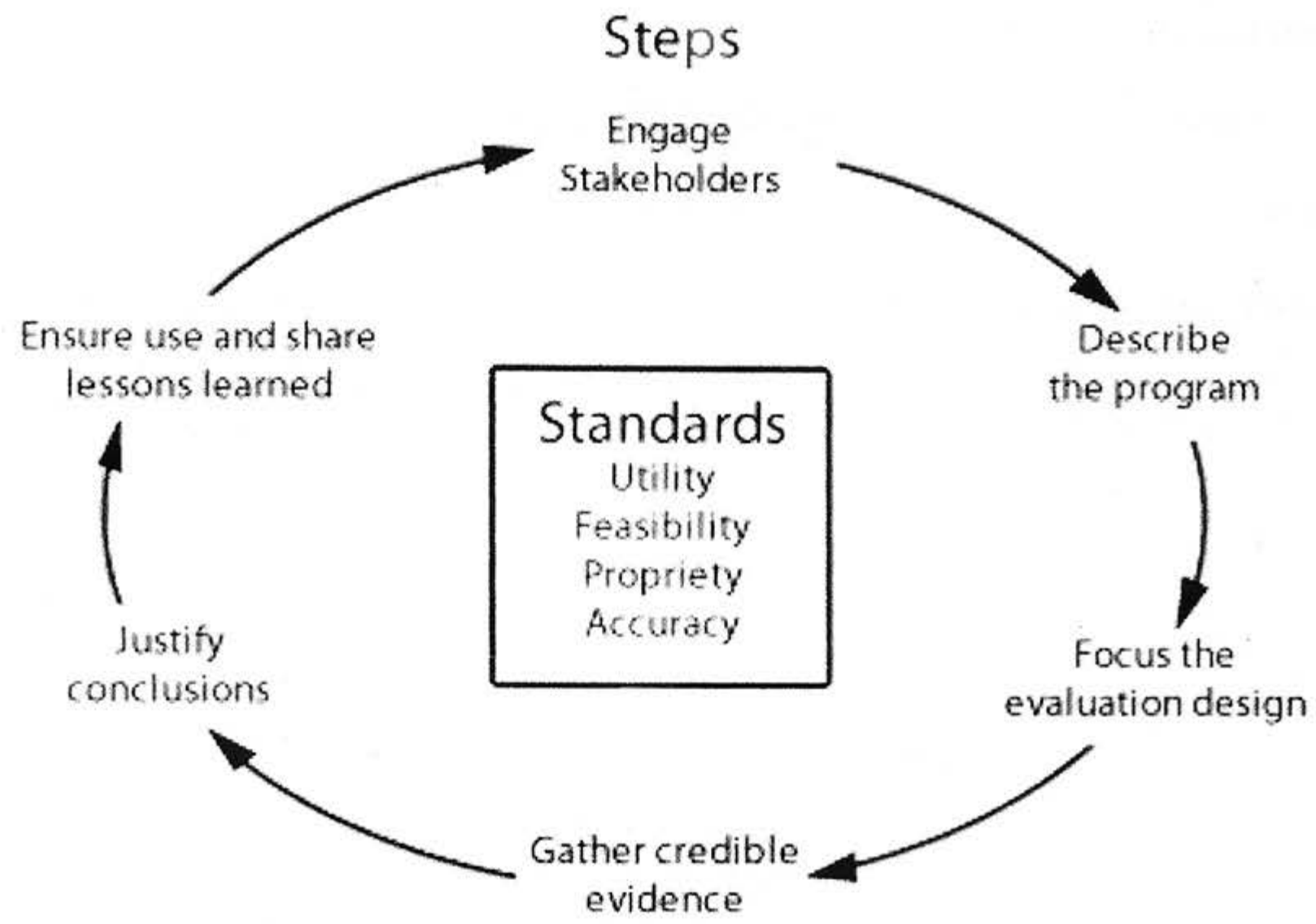

Figure 1: The CDC Framework for Program Evaluation

The CDC Framework consists of six steps and four standards that are used to evaluate the quality, value, and importance of a program and to bring to light areas for program improvement. The first step engages the stakeholders, or those who have an investment in what will be discovered in the evaluation and those involved in program operations. The values and concerns of those involved in the program should be understood. The next step describes the program in detail, including it's history and importance, its activities, expected effects, resources, and its context in the health care environment. The program goals are defined and agreed upon by the stakeholders. The third step focuses the evaluation design to include the issues of concern to the stakeholders, with a view towards utility and efficiency of resources. Factors to consider 
are the purpose, the users, the usefulness, the intended questions, the methods, and the agreements of those involved in carrying out the design. The next step is to gather evidence that strengthens the findings of the evaluation. Included in this evidence will be data about the effectiveness of the role and levels of client participation and satisfaction. A variety of sources should be sought in order to enhance the credibility of the evidence. The quality of the evidence and the limits of the quantity should be considered. The logistics involved in gathering the evidence must assure comfort and confidentiality for the participants. Step five is justifying the conclusions as they are linked to the evidence that has been gathered and the values held by the stakeholders. Analysis and synthesis, interpretation, judgements, and recommendations that are to be made will be clarified. Finally, step six is ensuring use of the work and sharing the lessons learned. This step includes providing feedback about interim findings to the stakeholders and planning for dissemination of the findings in advance. Evaluator credibility and impartial reporting is vital to this step. Design and planning for the presentation of findings, feedback and follow-up as needed are part of this step. Next, the methods used to conduct this evaluation will be discussed.

\section{Method}

\section{Purpose}

The purpose of this evaluation project was to assess the role of the NCM within the PCMH in order to determine the attributes, skills, practice protocols and resources which contribute to effectiveness of their role in the primary care setting. Secondarily, the attributes, skills and resources identified in the the literature were compared with the 
data obtained in interviews of NCMs in RI Primary Care practice group. This is a qualitative methadology where the data was collected through individual interviews of NCMs.

\section{Design}

A qualitative methadology was utilized and data were collected through individual interviews of $\mathrm{NCMs}$,

\section{Sample and Site}

The sample included was NCMs employed in the Rhode Island Primary Care Practice Corporation (RIPCPC) adult primary care practice groups in RI. RIPCPC has approximately 67 practice groups and they are currently transitioning all practices to PCMH according to the NCQA standards. At the time of this project,they had approximately $20 \mathrm{NCMs}$ working in these practices through a contract with Blue Cross/Blue Shield and NCMs were continuing to be hired. Permission to conduct the study was given from the clinical coordinator (Appendix A) and the project was approved by the RIC Institutional Revew Board (IRB). The desired sample size was 6-10 NCM participants. Pediatric PCMH practices were excluded because of the differences in morbidity and in patient outcomes for the pediatric population.

\section{Application of the CDC Framework}

The first evaluation step is to engage the stakeholders and ascertain their values and concerns. The focus of this project was the NCM role. However, in the process of securing permission to conduct the interviews, discussions with the physician coordinator and the Director of Clinical Quality provided significant background information. 
Next, a detailed description and an outline of the context for the creation of the NCM role in terms of quality health initiatives was discussed with the stakeholders. Then, credible evidence was gathered, including open-ended questioning of NCMs who responded to an invitational letter to participate in an interview. Careful analysis of findings, supported by sample comments and reviewed by the major advisor, were used to justify the conclusions drawn from the research literature and the interviews with the NCMs. The agreed upon standards must incorporate the values held by the stake holders or they will not use the results of the evaluation with confidence. Major themes from the interviews were summarized and clarified in order to be shared with the providers and the NCMs. The final step is to ensure use and share lessons learned. The steps include design of the dissemination of the results, preparation of the final document, feedback and follow-up. The results will be shared at a future educational meeting of the NCMs. Tools which are useful in chronic disease management such as online resources and educational opportunities will be compiled for use by the NCMs. There will be opportunities for questions and feedback at the meeting. Those who wish to follow-up by email with the author will be encouraged to do so. Throughout the evaluation, the author strove to adhere to the standards of feasibility, accuracy, propriety and utility set forth in the CDC framework for program evaluation.

\section{Measurement}

Interview questions were developed by this author based on the literature review as well as the clinical experience of the researcher and with input from the faculty advisor. Open-ended questions were used to obtain NCM descriptions of their role and 
their experiences working as a NCM. Questions were pilot tested with two NCMs who were not part of the sample. No voice recording was utilized, rather key words were recorded briefly on the data collection sheet (Appendix C).

The interview questions were:

1. Can you describe a typical day or client including assessments made, activities, etc.?

2. What skills did you use and what skills are needed to be effective?

3. What resources do you routinely use? Can you identify resources that might be needed?

4. What ways are you kept abreast of client changes and needs?

5. How do you think clients, physicians, and staff view your work?

6. Are there tasks or work you could be doing but are not doing now? Reason why not?

7. Are there tasks or work you do that could be delegated so you could spend more time with patients?

8. What was your education, experience, training, etc. for the job? Is it adequate?

9. Is there anything else that helps or hinders the role and your work?

10. Can you identify resources that you find most helpful in coaching patients?

11. What practice policies and advanced protocols do you find most effective in improving patient outcomes?

12. How do you determine health literacy and comprehension of self care management? 
13. How frequently are you informed of: a patients' visit to the Emergency Department; a patients' admission or discharge from the hospital? Do you have any suggestions for how continuity of care could be improved?

14. What are the biggest challenges you face in your practice?

15. Do you have adequate information technology support in tracking patient data and targeting those patients in need of information?

16. How would you like to see this role grow in the future and what would those changes require?

17. What else do you think would improve the quality of healthcare in our State?

\section{Procedures}

The RIC IRB procedures and approvals process was followed. A letter of permission from the physician who is the clinical coordinator of the NCMs in the RIPC practice group was obtained (Appendix A) as part of the IRB process. The investigator provided an invitational letter (Appendix B), approved and signed by the physician coordinator and the IRB, via RIPCPC email to NCMs employed in the RIPC practice group. NCMs were asked to participate in a brief interview. The investigator was contacted by potential participants and a meeting time and place was established. Before the start of the interview, the purpose of the study and what was expected of participants was described. Participants were provided with the consent document, and time was provided to review it. Participants were informed that interviews are anonymous and their individual responses would remain confidential. Participants were informed that interviews would not be tape recorded but rather that notes would be taken during the 
interview. However, their individual identity would not be recorded. Participants were reminded that participation was voluntary, and that they were free to withdraw their participation at any time without prejudice. Any additional questions were addressed at the time and informed consent was obtained prior to the interview (Appendix D). Interviews took place at RIPCPC main office and took 20-30 minutes to complete.

\section{Data Analysis.}

Data were de-identified, stored in a locked file, and data collection sheets were separated from the consents. Participants responses' were grouped by question. Then, themes were identified and grouped into eleven major categories. Lincoln and Guba established criteria for the reliability and validity of qualitative data (Polit, Beck, \&Hungler, 2001). Trustworthiness of the data can be evaluated by examining the credibility, dependability, confirmability, and transferability. In this case, credibility was established by prolonged engagement with the NCMs to establish trust and rapport. The NCMs were attending an educational collaborative day and were able to take time to meet with the interviewer. The data collection tool was used in the same way with each interviewee and notes were maintained. An external auditor also reviewed the notes which enhanced trustworthiness of the data.

\section{Results}

Eight NCMs participated in the interview process and responses were recorded on the data collection sheet developed by the researcher (Appendix C). 


\section{Findings}

The themes identified from the interviews with the eight NCMs can be organized into the following categories: professional skills; personal attributes; resources; practice policies; transitions of care coordination; intraoffice relationships; health literacy and teaching strategies; challenges; educational preparation; and patient response to the NCM. The first interview question was more informal in order to ease the transition into the questioning format. The NCMs were asked about a typical day including assessments made and activities performed. They were invited to share a success story about an intervention that contributed significantly to a patient becoming activated in selfmanagement of their illness. This was helpful in establishing rapport, and it became clear that these NCMs were passionate about their work with patients. Examples were offered about patients who had elevated fasting glucose and who normalized their blood glucose by taking steps to reduce their intake of carbohydrates and improve their activity levels. Another success story was a patient with COPD who was able to quit smoking with the support offered by the NCM.

Next, themes identified from the interview data will be presented.

Professional skills. One of the professional skills reported by the NCMs as necessary to be an effective NCM was health coaching using motivational interviewing skills in order to engage and activate the patient. Others skills identified by the NCMs were knowledge of chronic diseases processes, the ability to develop a plan of care with the patient, presenting information at the appropriate literacy level, and having the ability to see the patient within the context of their environment. 
Personal traits. Personal traits reported to be valuable included trustworthiness, flexibility, the ability to practice independently, excellent time management skills, commitment to problem solving, diplomacy, kindness, tactfulness, respectfulness, humility, confidence, and creativity. One comment was,"Meet the patients where they are and let them know that health behavior change is their choice." Another said "It is important to be flexible; see the patient in their home if they are not yet able to travel." Also "Speak plainly." The nurses seemed to understand intuitively that those they work with respond much more positively when not talked down to.

Resources. The NCMs identified online resources, training courses, the Director of Clinical Quality, and each other as resources. The NCMs varied in which resources they used in caring for patients. A list of online resources, called the Z-drive, is accessible to the NCMs on the practice group computer system. The 'Z-drive' is the place where most online resources are stored and is accessable to them in the field. Other online resources mentioned by NCMs included the ADA website, eClinicalworks, Epichart, and the National Lipid Society website. All of those interviewed mentioned the course offered by Blue Cross on motivational interviewing as an important resource. Another course mentioned by three of the NCMs was the guided care course offered by John Hopkins Medical School. One thought it was very good and two thought it was dated and not very helpful. The Department of Elderly Affairs was mentioned as a resource to help seniors with problems such as being unable to afford their prescriptions and other issues common to the elderly. The Good Neighbor Fund was utilized for those who needed heating assistance and the RI Food Bank for those who do not have enough 
money for food. Resources for patients who are diabetic included the ADA Bluebook, the Carb King carbohydrate counter, sparkpeople.com, and go meals which is an application that patients can use on their phones to manage their carbohydrate intake. The nurses indicated that they support one another by posting questions in the NCM mailbox and often get helpful and timely advice. The Director of Clinical Quality was mentioned by several of the NCMs as being very supportive and weekly meetings provide additional support.

Practice policies. The NCMs identified practice policies that were helpful to their work. Examples are ordering labs before patient visits, daily or weekly huddles with all staff to plan for the patients needs, and more timely communication between the staff, the physician and the NCM regarding patients who are having problems. Other practice policies that were found to be helpful were having the physician introduce them or let the patient know the NCM will be calling them. Managing anti-coagulation therapy on an Excel spread sheet and having a protocol for glucose pattern management enabled the NCMs to assist patients more efficiently. Starting a patient on a new medication in the office using samples was found to help increase adherence.

Transitions of care coordination. Continuity of care is often interupted when a patient is admitted or discharged from a facility. The average rate of timely notification of admission or discharge was thought to be a little over $40 \%$ according to the NCMs. The area of transitions of care is being targeted as an important one because patients need the most help at that time in order to reduce medication errors and readmissions to healthcare facilities. Lifespan hospitals were rated as better than most at notifying 
primary care providers although Kent Hospital was thought to be improving. Often, the nurse reported gettting the list from Blue Cross three or four weeks after a patient is discharged from the hospital. Strategies for improvement include timely faxes to the physicians from urgent care centers, emergency departments, and hospitals and other healthcare facilities. Also, improved nurse to nurse communication from visiting nurses and hospital case managers was suggested.

Intraoffice relationships. The NCMs reported some struggles in their relationships with the medical assistants and office managers. In one case, it was reported the medical assistant seemed resentful of the NCM when reminded of the importance of checking the patients height in order to calculate their BMI. One said "It is important to help the medical assistants understand the why of PCMH and to let them know their role is important." Also "Be positive, let them know you are there to help." In most cases, though, the NCMs described a gradual building of rapport with the medical assistants and office managers built on mutual respect and trust. The NCMs reported generally positive relationships with the physicians in the practice. However, one NCM thought the physician was too willing to give the NCM the most difficult and non-compliant patients.

Health literacy and teaching strategies. The question on determining health literacy and teaching strategies yielded rich data. The responses included asking the patient what they know about their diagnosis and what they like to read. Another mentioned asking the patients questions such as "What will you tell your family about your diagnosis?" and "What will you do differently at the grocery store or at a 
restaurant?" One NCM said that she tells her patient "You are not a diabetic, you are a man who has diabetes." If a patient is tech-savvy, the use of apps such as 'go meals', Sparkpeople and others were mentioned. These responses really highlighted the importance of having expert nurses in the role of the NCM to assess health literacy and select appropriate teaching strategies.

Challenges. The NCMs reported many challenges in carrying out their responsibilities. One was the need for more behavioral health services support. One NCM suggested that all patients with chronic diseases be more carefully screened for depression. Two NCMs mentioned an inadequate amount of tech support and a condescending attitude from some of the IT staff. Others issues were working with physicians who do not understand the role of the NCM. Also, there were the challenges of encouraging the practices to meet NCQA guidelines for PCMH and to focus on Meaningful Use (MU), which is mandated for Medicare and Medicaid reimbursement, and improving outcomes. Other concerns noted were the high number of practices and patients to see. Also mentioned was the lack of an accurate risk stratification on the utilization list from Blue Cross. Sometimes patients on the list were low risk and did not need a visit while the high risk patients were left off the list leading to inefficiencies in patient interventions.

Educational preparation and experience. The NCMs had varying educational preparation and experience. Three of the NCMs were Cerified Outpatient Diabetic Educators (CDOE). Of the eight interviewed, there were two NCMs with an Associates Degree in Nursing, one with a Diploma in Nursing, one with a Baccalareate in Nursing 
and a Masters in Social Work and four had Baccalaureate Degrees in Nursing. The number of years of nursing experience varied, but all had at least 15 years of nursing experience. The NCMs were all expert nurses with an apparent love of learning.

Patient response to NCM role. When asked how they perceived the patients reaction to them in their role, the NCMs indicated that it depended somewhat on the introduction. One said "If the patients felt bullied into seeing the NCM, it was a rough start." In most cases, after initial skepticism and a greater understanding of the role, the patients expressed gratitude for the teaching and follow-up provided.

Physician referrrals. Physician resistance to referring patients was noted by one of the sample of NCMs, but most thought the physicians were consistent in delegating patients who needed case management.

Future growth of the role of the NCM. The NCMs unanimously support the growth of the role of the NCM as the key to driving down costs. They would like to spend less time on NCQA requirements and more time on patient interactions. One NCM stated "When the physicians embrace the spirit of PCMH, the outcomes will improve." She predicts this will have a huge impact on patient care and healthcare costs in our state.

\section{Dissemination}

The results will be shared and ideas discussed in one of the NCMs educational meetings; tentatively scheduled for late spring. The presentation will include the major themes identified from the literature and the interviews will be summarized and discussed. A list of the identified helpful tools and resources as well as additional 
resources identified by the researcher will be offered for use by the NCMs. Results will also be shared with the physicians and other key members of the RIPC practice group at that time.

\section{Summary and Conclusions}

Nurse case management for those with chronic diseases yields improved patient outcomes that can translate into cost savings (Wilson et al., 2005). The purpose of this evaluation project was to assess the role of the NCM within the PCMH in order to determine the attributes, skills, practice protocols and resources which contribute to effectiveness of their role in the primary care setting. Eight NCMs within the RIPCPC practice group agreed to participate and completed the qualitative interview format.

Thematic analysis identified the following themes from the data: professional skills; personal attributes; resources,; practice policies, transitions of care coordination, intraoffice relationships, health literacy and teaching strategies, challenges, educational preparation, and patient response to the NCM.

Several attributes and interventions by the NCMs were associated with improved patient outcomes in the literature. These can be organized into three categories: Role, Interventions, and Attributes.

Role

Improved tracking of transitions of care is associated with a decrease in readmissions to hospitals because of additional patient support from NCMs during critical periods (Managed Care, 2011). By definition, the role of NCM role is to reduce fragmentation and evaluate options available to help meet a patient's health needs 
through collaboration with the patient and physician in order to achieve improved quality of life (Case Management Society of America, 2002). The role of the NCM is in line with the IHI's triple aim for healthcare: to improve population health, enhance the patient experience and reduce per capita cost (Managed Care, 2011).

This was consistent with the role identified by the NCMs. They described the coordination of patient care and follow-up after hospital discharges. They also discussed some of the delays and difficulties related to the follow-up after hospital discharges. The NCMs also identified the value of health coaching and the personal traits they believed were associated with effectiveness in the role. Some role confusion among office staff and some doctors was reported but they stated it was not a major problem. Also they commented that if the patient was bullied into seeing the NCM then the patient was not as accepting of the role. However, most patients were accepting of the NCM. The NCMs identified their Director of Clinical Quality, who holds weekly meetings with the NCMs and their colleagues, to be very helpful to them in their role.

\section{Interventions}

The literature lists several interventions. The use of MI and the transtheoretical model enhances patients engagement in care, encourages goal setting, and leads to more activated patients (Dellsaga C., 2010). The use of EMR to track patients who are highrisk and the use of clinical decision support tools in the EHR helps to ensure that patients are receiving optimal care for their conditions (Bowers, 2010). Studies about the effectiveness of NCMs listed interventions such as creating self-care management plans, ensuring patients made appointment and tracking lab work. The interviews with the 
NCM also identified the use of MI, health coaching, and self management education. Another area NCMs discussed was referrals of patients to community resources. They described how they use their computer system to maintain a database of community resources. They also described how they used the resource list to help meet patient needs identified during their interviews.

\section{Attributes}

The literature highlighted several elements of NCM practice. Practices leading to improved patient results include collaboration among clinicians (Welch, 2010), specific training for NCMs, and advanced protocols (Aubert, 1998; Sutherland, 2009; and Watts S, 2011). Evidence based practices such as shared medical appointments (Watts, 2009), and addressing the social context of the patient (Mullen, 2006) were important in the literature. The advantage of expert and experienced nurses (Sutherland, 2009) who have excellent communication skills (Genrich, 2001) was emphasized. The need for educational opportunities, particularly in case management for those with chronic diseases was underscored (Genrich, 2001). Case management requires specific skills and it was suggested that new curriculums be developed to ensure role clarification and skill development of NCMs (Genrich, 2001).

The interviews with the NCMs matched many of these same practice areas. Professional skills the NCMs identified were health coaching, using motivational interviewing skills, knowledge of chronic diseases processes, the ability to develop a plan of care with the patient, presenting information at the appropriate literacy level, and having the ability to see the patient within the context of their environment. Personal 
traits included trustworthiness, flexibility, the ability to practice independently, excellent time management skills, commitment to problem solving, diplomacy, kindness, tactfulness, respectfulness, humility, confidence, and creativity. Their educational background and certifications varied but all had at least 15 years of nursing experience. The NCMs were all expert nurses with an apparent love of learning.

\section{Limitations.}

Limitations of this program evaluation were that because $\mathrm{PCMH}$ is a relatively new model, there was a scarcity of literature on the role of the NCM in PCMH. However, the articles on chronic disease case management were useful and the consistency between the literature and the qualitative data suggests that the role is evolving successfully. Additionally, the interviews lacked diversity as all the NCMs were Caucasian women.

Next, recommendations and implications will be discussed.

\section{Recommendations and Implications}

Recommendations for growth of the role of the NCM include engaging patients, use of technology, collaboration, practice policies and operations, education, and future research.

\section{Engaging Patients}

The trend toward an activated patient who takes responsibility for his/her own health and who demands more timely access to care is both optimistic and realistic. We are in the midst of a nationwide focus on healthy lifestyles and patients are looking for a proactive emphasis on prevention from their health care providers. Patient portals are 
opening up the channels of communication with providers, and patients want to be informed about their test results, get information about health topics, and make their own appointments. The NCM in a PCMH is ideally suited to work with patients and engage them in self management of their chronic diseases. The health coaching, motivational interviewing, monitoring health measures and referrals to resources are active interventions that encourage engagement by and with the patients. Sending a letter to high-risk patients in the practice to inform them about the role of the NCM would help to clarify the role and explain the connection between the NCM and the patient's physician and also validates the importance of the NCM's role in the PCMH practice.

\section{Use of Technology}

EHR needs greater interoperability in order to maximize MU initiatives and expedite the communication between facilities and PCMH practices. Only pertinent patient information should be sent to make the transfer of information less cumbersome. Improving continuity of care could also be accomplished by providing increased incentives to facilities that consistently notify PCPs of patient treatment. The use of technology to improve communication between facilities, community based clinicians such as home care nurses, and the primary care offices would have a tremendous impact.

\section{Collaboration}

Collaboration among health professionals, patient, and family members is the future of care in a PCMH. Seeking patient feedback about his/her care in order to direct quality improvements and involving family members in shared medical appointments featuring collaboration among clinicians are part of the future of care in a PCMH. 
Teamworks is a RI DOH program that practices can participate in. These visits feature a shared medical appointment model in which the physician, a nurse diabetic or cardiovascular educator, and a dietician all meet with a group of patients in an educational forum before the visit with the physician. A pharmacist is available to review medications with the patients and suggest generic alternatives to the physician as indicated. Use of this and other similar models is recommended.

\section{Practice Policies and Operations}

Although the NCMs interviewed were an accepted part of the practice, there were office procedures and operations needed to facilitate the work by the NCM. PCMH practices that strive for timely phone response and have allotted room in the schedule for sick visits help to decrease over-utilization of emergency departments and urgent care facilities. Additional time allotted for pre-visit planning would help to improve patient outcomes. A review of the schedule of patients by the staff on a weekly or daily basis would help to plan for patient needs for preventative screenings, laboratory work, or referrals to support staff. Referrals to the nutritionist, behavioral health support, and pain clinics as needed all contribute to optimal patient outcomes.

\section{Education}

Education that helps all staff to work to maximize their potential is the goal of one of the RIPCPC physicians. NCMs can provide educational opportunities to medical assistants that raise the level of professional integrity and contribute to their development. NCMs can take advantage of educational offerings such as Diabetes, Asthma, and Cardiovascular Outpatient Educator classes. Becoming certified as a case manager or a 
smoking cessation counselor are other ideas for advancing the education of NCMs. All clinicians should be able to use MI with confidence in order to help patients to set their health goals.

Graduate education in community or public health is also recommended. The demands of the role include evaluative research, the ability to communicate with diverse groups, the ability to resolve conflicts, and knowledge of economic realities. Specific curriculums to address the current needs of case management have been developed in baccalaureate and graduate programs (Genrich, 2001).

\section{Public Policy}

The burden of chronic disease on our medical system and on society is substantial and adds to the difficulty of managing ever-increasing amounts of patient information as care becomes more complex. It has placed the quality of health care in the United States (US) under scrutiny because of medical errors, lack of coordination, patient frustration and poor outcomes. In response to the many challenges, the Patient-Centered Medical Home $(\mathrm{PCMH})$ model of care was developed and is designed to increase the value of our health care and to improve the experience for the patient. The role of the Nurse Care Manager (NCM) is a key element in the PCMH. As pay for performance becomes more widespread, the skills and effectiveness of the NCM in disease management and health promotion programs will become even more important.

\section{Future Research}

As mentioned earlier there is limited research about NCMs and in particular NCMs in a PCMH. There are many research questions to be investigated. For example: 
How can we best address the social determinants and environmental context of patients lives in order to determine why some patients are more proactive than others?; What are their supports and stressors?; What would help nurses to manage chronic disease more effectively in diverse populations?; What kind of incentives would help to activate and engage patients who are fatalistic or reluctant to make behavioral changes? And last, How can team performance best be evaluated given the variation in practice demographics and ethnic groups?

\section{Conclusion}

The role of the NCM in PCMH seems to be assured. Critical thinking, clinical expertise, and good communication skills are the foundation for the many attributes of an effective NCM. This study affirmed the role of the NCM is evolving sucessfully in response to the need to improve the value of health care. Having a NCM in the practice to offer self-management support, coordination of care, and track patient outcomes is an assential part of an NCQA certified PCMH. Chronic diseases such as heart disease, diabetes, asthma, obesity, hypertension, chronic renal failure, and COPD affect $20 \%$ of the population and account for $75 \%$ of health care spending (Wu, 2000). This number is expected to rise as our population ages. As pay for performance becomes more widespread, disease management and health promotion programs will become even more important. NCMs are effective in assisting patients in managing chronic disease, engaging and activating the patient, transition of care management, and using electronic medical records (EMR) to track patient outcomes. Collaboration with other clinicians, evidence-based and advanced protocol interventions, advanced education and training, 
and understanding the social and environmental context of the patient were all found to improve patient care. The above recommendations can be used to add to the description of the role for new NCMs and point the way for future growth of the role of the NCM in PCMH. 


\section{References}

American Nurses Association. (2001). Nurse case management. Kansas City.

Aubert, R. W. (1998, October 15). Nurse case management to improve glycemic control in diabetic patients in a health maintenance organization: A randomized, controlled trial. Annals of Internal Medicine , 605-612.

Bohmer, R. \&Lee,T (2009). The shifting mission of health care delivery organizations. New England Journal of Medicine , 361 (6), 1-6.

Bowers, J. (2010, September). Rhode Island practice adopts patient -centered medical home. ACP Internist .

Brodie, D.,Inoue, A., and Shaw, D. A. (2006). Motivational Interviewing to change quality of life for people with chronic heart failure; A randomised, controlled trial. International journal of Nursing Studies , 489-500.

Case Management Society of America. (2002). Membership information. Retrieved 2011, from http://www.cmsa.org/meminfo/mem

Centers for Disease Control and Prevention. (2011, August 3). CDC-Program Evaluation Steps. Retrieved August 11, 2011 from CDC: http://www.cdc.gov/eval/steps/index.htm

Dellsaga C., Gabbay, R.,Durdock, K., and Matinez-King A.- (2010). Motivational interviewing to change type 2 self-care behaviors. Journal of Diabetes Nursing , $14(3), 112-118$. 
Genrich, S. Neatherlin, J. (2001). Case manager role: A content analysis of published literature. Case Management Journals , 14-19.

Homer, C., and Baron, R. (2010). How to scale up primary care transformation: What we know and what we need to know. Journal of General Internal Medicine, 625-9.

Institute of Medicine. (2001). Crossing the quality chasm: A new health system for the 21st century. Washington,DC: The National Academy Press.

Institute of Medicine. (2006). Rewarding provider performance: Aligning incentives in medicare. Washington, DC: The National Academies Press.

Jonas S., Kovner, A. and Knickman,J. (2008). Overview: The state of healthcare delivery in the united states. In Healthcare Delivery in the United States (pp. 18-24). NY, NY: Springer Publishing Company.

Managed Care. (2011, April). Geisinger's embedded nurses improve transitions. Managed Care , 20 (4).

Mullen, B. Kelley, P. (2006). Diabetes nurse case management An effective tool. American Academy of Nurse Practitioners , 22-30.

Patient-Centered Medical Home. (2011). Retrieved July 6, 2011 from NCQA: http://www.ncqa.org/tabid/631

Patient-Centered Primary Care Collaborative. (2010, November 16). PCMH-Evidence of quality. Retrieved September 12, 2011 from http://www.pcpcc.net/content/pcmh-outcome-evidence-quality 
Polit, DF., Beck, CT., and Hungler, BP. (2001) Essentials of nursing research, methods, appraisal, and utilization Philadelphia, Pa. Lippincott, Williams \& Hungler Rollnick, S., Mason P. (1999). Health behavior change: A guide for practitioners. Edinburgh: Churchill Livingstone.

Sutherland, D., Hayter,M. (2009). Structured review:evaluating the efectiveness of nurse case managers in improving health outcomes in three major chronic diseases. Journal of Clinical Nursing ,2978-2992.

The PCMH Advisory Committee. (2011). NCQA's patient-centered medical home 2011. US Department of Health and Human Services. (2010). National partnership for action to end health disparities. Retrieved March 2011 from NPA: htttp://minorityhealth.hhs.gov/npa/templates

Watts S, Lawrence, R., and Kern E (2011, January 11). Diabetes nurse case management training program: enhancing care consistent with the chronic care and patient centered medical home models. Clinical Diabetes , 29 (1), pp. 25-33.

Watts, S. Gee, J., O'Day, M., Schaub, Lawrence, R., Aron, D., and Kirsh, S. (2009). Nurse practitioner-led multidisciplinary teams to improve chronic illness care: The unique strengths of nurse practitioners applied to shared medical appointments/group visits. Journal of the American Academy of Nurse Practitioners, 167-172.

Welch, G,. Garb, J., Zagarins, S., Lendel, I., and Gabbay, R. (2010). Nurse diabetes case management interventions and blood glucose control: Results of a meta-analysis. Diabetes Research and Clinical Practice , 1-5. 
Wilson C., Curtis, J., Lipke, S., Bochensky, C., and Gilliland, S. (2005). Nurse case manager effectiveness and case load in a large clinical practice: implications for workforce development. Diabetes Medicine , 22 (8), 1116-20.

Wu, S., Green, A. Projection of chronic illness and cost inflation (2000) RAND Corporation 


\title{
Appendices
}

\section{Appendix A: Letter of Permission}

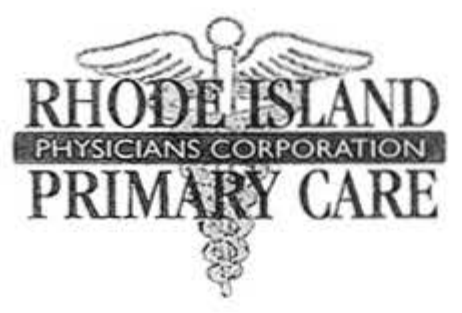

Dear RIC IRB Board Members:

December $13^{\text {th }}, 2011$

\begin{abstract}
I have had a discussion with Susan Corner about the Nurse Care Manager Role Evaluation Project being conducted this year with Rhode Island Primary Care Physician's Corporation (RIPCPC) practice group, Hillside Avenue Family \& Community Medicine being one of the participating Practices. I understand the scope of the project and I have been informed that interviews with individual nurse care managers (NCM) will be conducted after informed consent is obtained. As a family practice physician who has worked with many RI primary care practices to facilitate the transformation to a patient centered medical home, I very much appreciate the vital role being played by the new position of nurse care manager. I give my permission for the interviews to take place at the worksite or another convenient location. I feel a further assessment of $\mathrm{NCM}$ work at this time will be useful in understanding how well this position is being utilized in the primary care setting and will contribute to maximizing the impact of this pivotal position.
\end{abstract}

Sincerely,

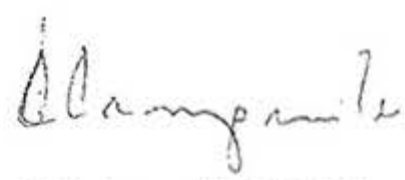

Chris Campanile, MD PhD

Hillside Avenue Family \& Community Medicine

Clinical Coordinator, Healthcentric Advisors (formerly Quality Partners of Rl)

Clinical Associate Professor, Department of Family Medicine, Warren Alpert Medical School, Brown University 
Appendix B: Invitational Letter to NCM's

February 1,2012

Hello,

My name is Susan Corner and I am interested in understanding the role of the Nurse Care Manager within the Patient Centered Medical Home. The purpose of this research project is to clarify the NCM role as it evolves and to add to the file of helpful resources and educational opportunities in chronic disease management used by the NCMs. This work represents my final project as a Master of Science in Nursing student at Rhode Island College. Your help would be very much appreciated. I am sending this letter to invite you to assist in this research if you are interested.

If you decided to participate, you would be asked to complete an interview of open-ended questions that would take approximately $30-45$ minutes to complete. Your responses will remain anonymous and confidential, and will not be tape-recorded. If you decide to participate, you may withdraw your participation at any time.

If you are available to take part in this project, please contact me at scorner_1910@email.ric.edu or by phone@ 248-4326.

Thank you, Susan Corner, RN 


\section{Appendix C: Data Collection Tool}

1. Can you describe a typical day or client including assessments made, activities, etc.?

2. What skills did you use and what skills are needed to be effective?

3. What resources do you routinely use? Can you identify resources that might be needed?

4. What ways are you kept abreast of client changes and needs?

5. How do you think clients, physicians, and staff view your work?

6. Are there tasks or work you could be doing but are not doing now? Reason why not?

7. Are there tasks or work you do that could be delegated so you could spend more time with patients?

8. What was your education, experience, training, etc. for the job? Is it adequate?

9. Is there anything else that helps or hinders the role and your work?

10. Can you identify resources that you find most helpful in coaching patients?

11. What practice policies and advanced protocols do you find most effective in improving patient outcomes?

12. How do you determine health literacy and comprehension of self care management?

13. How frequently are you informed of: a patients' visit to the Emergency Department; a patients' admission or discharge from the hospital? Do you have any suggestions for how continuity of care could be improved?

14. What are the biggest challenges you face in your practice?

15. Do you have adequate information technology support in tracking patient data and targeting those patients in need of information?

16. How would you like to see this role grow in the future and what would those changes require?

17. What else do you think would improve the quality of healthcare in our State? 


\section{Appendix D \\ CONSENT DOCUMENT \\ Rhode Island College \\ Nurse Case Managers and Patient Centered Medical Home}

You are being asked to participate in a research study about the role of the nurse care manager in Patient Centered Medical Home. You were selected as a possible participant because you are employed as a nurse care manager. Please read this form and ask any questions that you may have before agreeing to be in the research.

Susan Corner, RN, CDOE, a Master of Science in Nursing student at Rhode Island College is conducting this study.

\section{Background Information}

The purpose of this evaluation project is to assess the role of the NCM within the PCMH in order to determine the best practices and resources which contribute to effectiveness of their role in the primary care setting. The secondary purpose is to compile a toolkit of resources, practice policies, and educational opportunities which may help the NCM to do their job more successfully.

\section{Procedures}

If you agree to be a participant in this research, you will be asked to do the following things: to meet with the investigator to participate in an interview with several open ended questions exploring the role of the NCM. The interview will take approximately 30-45 minutes to complete Time for additional comments will be provided.

\section{Risks of Being in the Study}

The risks of participating in this research are minimal, meaning that they are about the same as what you would experience in your normal daily activities. You may ask to stop the interview if it becomes burdensome in any way.

\section{Benefits to You}

Benefits to the participants are the opportunity to articulate and clarify your thoughts about the role of the NCM and to give your opinions about the future of the role of the NCM in PCMH. Your responses will remain anonymous and confidentiality will be assured. You will also be provided a list of resources and tools for use in the management of patients with chronic diseases after this project is completed.

\section{Voluntary Participation}

Your participation is completely voluntary. Your employers do not require it. You can choose not to participate in this research and it will have no effect on your employment. Also, you can change your mind about participating at any time with no negative consequences.

Initial here to indicate that you have read and understood this page. 
The records of this research will be kept private. In any sort of report that might be published, the researcher will not include any information that will make it possible to identify you. Research records will be kept in a secured file, and access will be limited to the researcher. If there are problems with the study, the research records may be viewed by Rhode Island College review board responsible for protecting human participants and other government agencies that protect human participants in research. All data will be kept for a minimum of three years, after which it will be destroyed.

\section{Contacts and Questions}

The researcher conducting this study is Susan Corner. You may ask any questions you have now. If you have any questions later, you may contact her at scorner_1910@email.ric.edu or 401-248-4326. The student advisor is kgremel@ric.edu.

If you think you were treated unfairly or would like to talk to someone other than the researcher about your rights or safety as a research participant, please contact Dr. Christine Marco, Chair of the Rhode Island College Institutional Review Board at IRB@ric.edu, or by phone at 401-456-8598, or by writing to Dr. Christine Marco, Chair IRB; c/o Department of Psychology, Horace Mann Hall 311; Rhode Island College; 600 Mount Pleasant Avenue Providence, RI 02908.

You will be given a copy of this form for your records.

\section{Statement of Consent}

I have read and understand the above information, and I agree to participate in the study "Nurse Case Managers and Patient-Centered Medical Home"I understand that my participation is voluntary and can be withdrawn at any time with no negative consequences. I have received answers to the questions I asked, or I will contact the researcher with any future questions that arise. I am at least 18 years of age.

Print Name of Participant:

Signature of Participant: Date:

Name of Researcher Obtaining Consent: 
Appendix E

\section{Additional Resources for NCMs}

Information for patients:

Medline Plus.gov .

Up-to-Date online

For Diabetics:

Myplate.gov

Learning about diabetes.org

Ndep.nih.gov/publications (recipes for Hispanic patients)

Sparkpeople.com (online tracking of nutrition and activity)

University of Michigan-the Great Plate: MHealthy.umich.edu

ACP and ADA: Managing the ABC's of Diabetes

For patients with COPD:

The National Heart Lung and Blood Institute: Breathing Better with a COPD Diagnosis www.nhlbi.nih.gov/health/public/lung/copd

American Lung Association: State of Lung Disease in Diverse Communities 2010

www.lung.org/your-lungs/protecting-your-lungs/

Management tools:

The American Academy of Family Physicians toolkit-

www .aafp.org/online/en/home/publications/journals/fpm/fpmtoolbox.html.

The Institute for Healthcare Improvement: www ihi.org/IHI/Topics/ChronicConditions/.

RI DOH Initiative for a Healthy Weight: Eat Smart, Move More: Plan for Action 2010-2015www.health.ri.gov/publications/actionplans/2010InitiativeForHealthyWeight.pdf

National Institutes of Health Diabetes Prevention Plan:www.bsc.gwu./dpp/manuals.htmlvdoc 